\title{
Online Supplement to "Time series analysis of real-time music perception: approaches to the assessment of individual and expertise differences in perception of expressed affect"
}

\author{
Roger T. Dean ${ }^{\mathrm{a} *}$, Freya Bailes ${ }^{\mathrm{a}, \mathrm{c}}$, and William T.M. Dunsmuir ${ }^{\mathrm{b}}$ \\ ${ }^{a}$ MARCS Institute, University of Western Sydney, Sydney, Australia; \\ ${ }^{\mathrm{b}}$ Department of Statistics, University of New South Wales, Sydney, Australia; \\ ${ }^{\mathrm{c}}$ School of Drama, Music and Screen, University of Hull, Hull, UK
}

(v3 released October 23, 2014)

\begin{abstract}
This Online Supplement material details the general linear autoregressive moving average (GLARMA) models we have developed to assess whether the results of the conventional ARMA and other time series analysis models in the paper can be confirmed. This arose because the common occurrence of significant periods of stasis during the response series might question some assumptions of conventional time series analysis. The results here are consistent with those from the conventional time series analysis, confirming its continued applicability.
\end{abstract}

Keywords: music; time series analysis; continuous response analysis; GLARMA

\section{Binary analyses of individual response time series: a solution to the "perceived arousal stasis" conundrum}

In Section 5 of the paper we presented results from modelling individual responses using the standard ARMAX time series modelling approach. However, as is clear from Figure 1 , listeners differ substantially in the amount of time that their responses are flat, that is, showing no change in successive time points: $15 \%$ to $90 \%$ of time points over the 32 listeners. The question that we pursue here, detailing the information summarised in the corresponding section of the main paper, is the extent to which the observed periods of stasis impact on the conclusions obtained from applying traditional time series methodology, designed for response series which have a continuous distribution. For this we develop an approach based on component series and binary time series modelling. Before explaining the approach, we consider in more detail the results of the standard ARMAX model discussed in the main paper for the four selected listeners 5 EA, $13 \mathrm{M}$, 23 NM, and 22 NM shown in Figure 1.

\footnotetext{
*Corresponding author. Email: Roger.Dean@uws.edu.au
} 
Listener 5 EA

$(\%$ Flat $=15)$

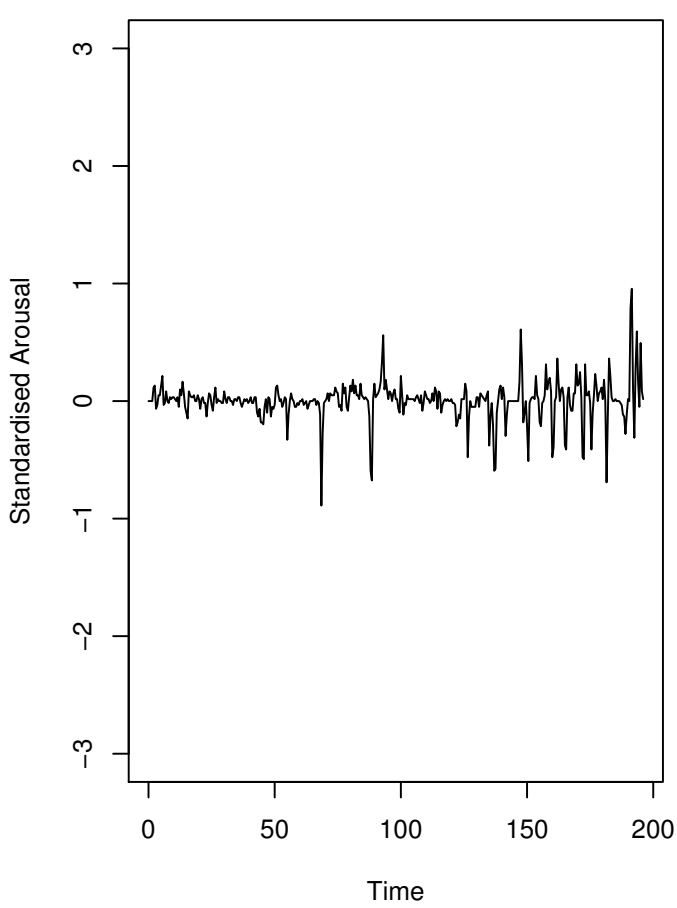

Listener $23 \mathrm{NM}$

$(\%$ Flat = 52 )

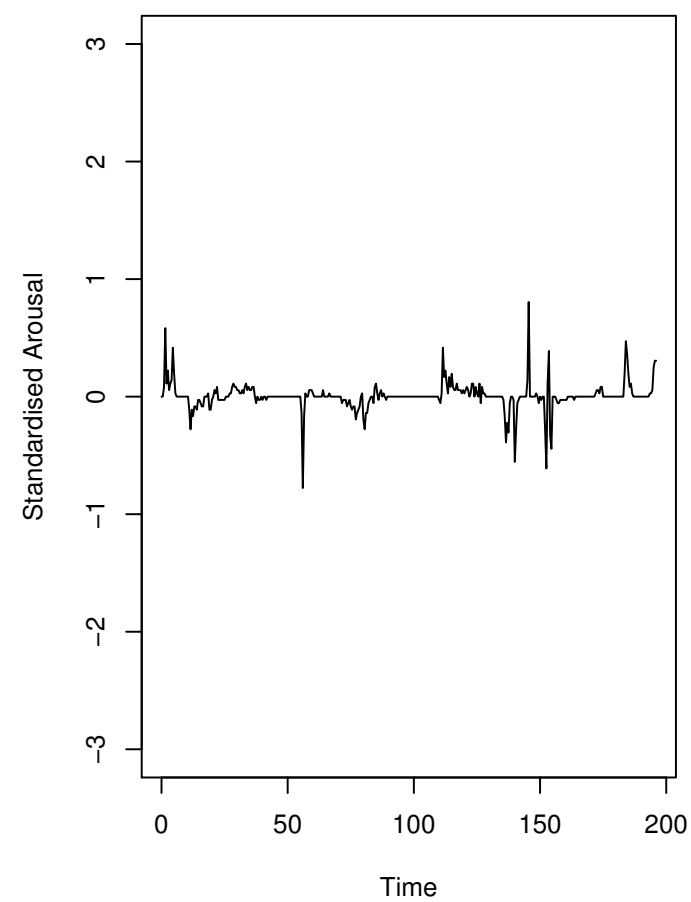

Listener $13 \mathrm{M}$

$(\%$ Flat $=30)$

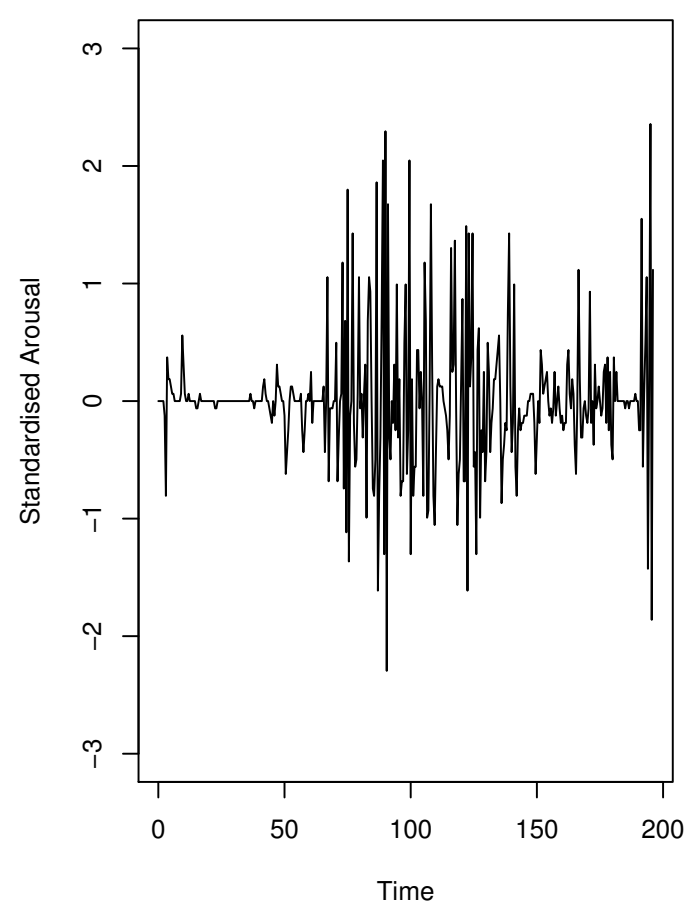

Listener $22 \mathrm{NM}$

$(\%$ Flat $=88)$

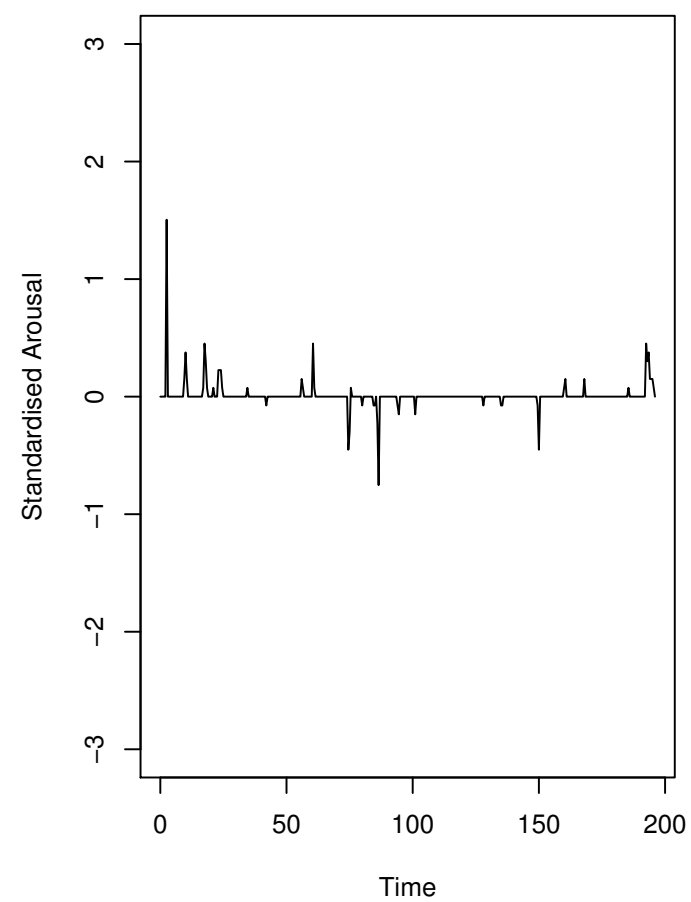

Figure 1. Lag 1 differenced standardised arousal series for four listeners. Percentage of times that the difference is zero is shown as \% Flat. 

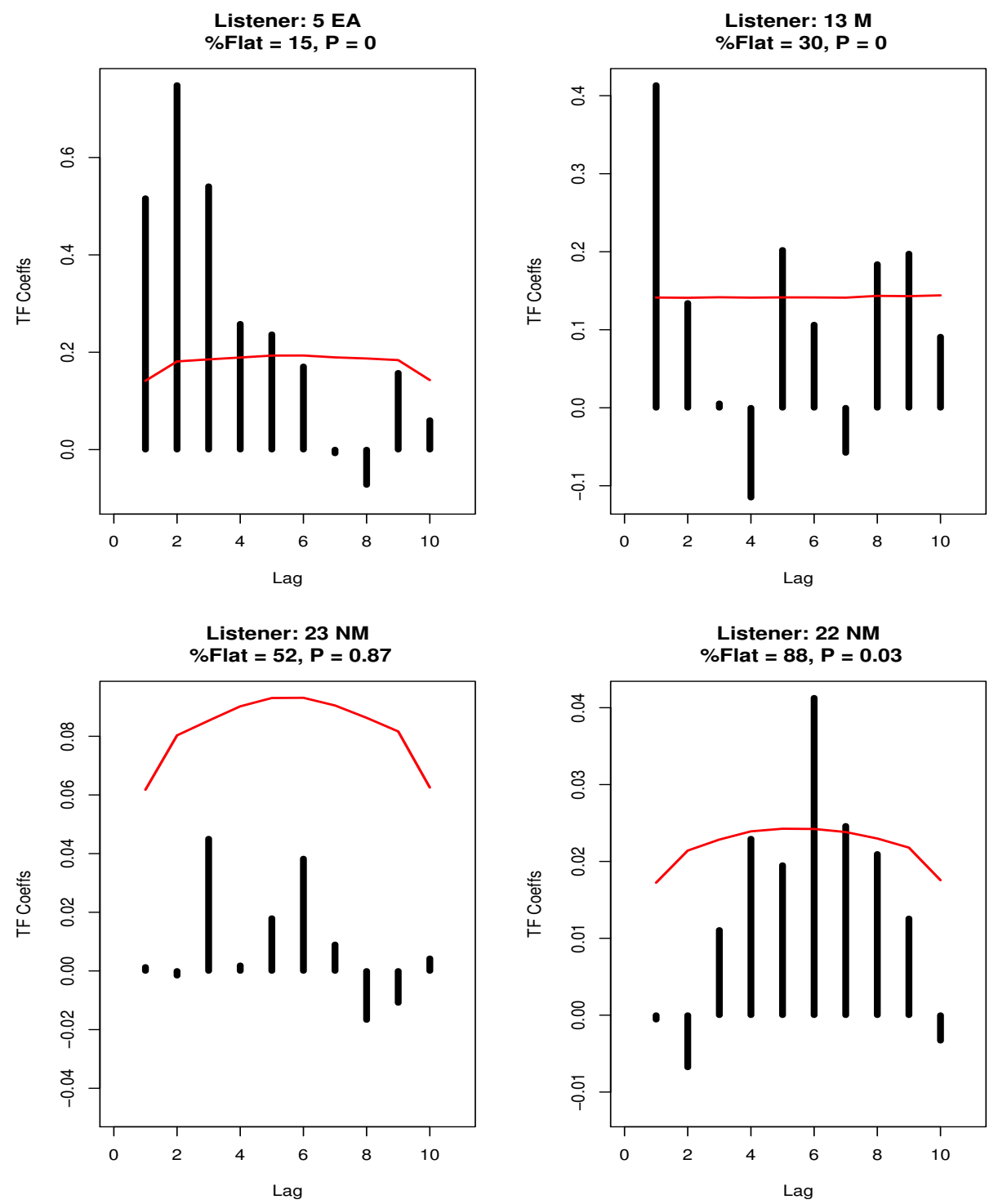

Figure 2. Transfer Function Coefficients Using an ARMAX model with 10 lags for four selected cases (1.96× standard errors for individual coefficients are shown as the red line.)

The fitted ARMAX model transfer function coefficients and their individual $1.96 \times$ s.e. limits for these four series are shown in Figure 2. The $p$-value shown in the titles is for the Wald test of overall significance of the transfer function. Listeners 5, 13 and 22 show significant responses overall as well as having individually significant transfer function coefficients. Listener 5 has a reasonably regular pattern of transfer function coefficients with a peak at lag 2 and a decay thereafter. Listener 13 has a peak transfer function at lag 1 and then somewhat sporadic significant lags. Listener 22 shows a reasonably smooth ascendancy to a peak value at lag 6 and then a decay thereafter. Thus individuals vary in the level of overall significance of their transfer function, and given significance, the lag at which they exhibit peak response varies from early to middle to late.

In the remainder of this Online Supplement we develop an alternative model which explicitly accounts for the highly discrete nature of the individual response series during periods of stasis. We compare the results from this alternative approach with the earlier results for the four selected series just discussed. We also apply the alternative approach to all 32 individual response series. We use the binary model to re-examine the question of 
differences between groups and individuals and whether any differences can be explained by other factors as we did in the main body of the paper.

\subsection{A model for binary component series.}

Our alternative approach to the traditional transfer function models of the main paper is to model the series of lag 1 differences in arousal response with two components. The first component would denote the sign of the change where, for the $j$ th listener at time $t$,

$$
D_{j, t}=\operatorname{sign}\left(\nabla Y_{j, t}\right)=\left\{\begin{array}{c}
-1, \text { if } \nabla Y_{j, t}<0 \\
0, \text { if } \nabla Y_{j, t}=0 \\
1, \text { if } \nabla Y_{j, t}>0
\end{array}\right.
$$

and the second component could be the size $\left|\nabla Y_{j, t}\right|$ of the change at times where $D_{j, t} \neq 0$. Thus we might model $\nabla Y_{j, t}$ in terms of a model for the trinomial process $D_{j, t}$ and, conditional on this process being non-zero, model the size of the change in arousal response. This approach has been successfully applied in modelling high frequency financial data - see for example (Rydberg and Shephard 2003) for a model which uses two binary processes and (Liesenfeld, Nolte, and Pohlmeier 2006) for a model using the trinomial observations. We take a binary focus, modelling positive changes as contrasted with null or negative changes, as a highly conservative and stringent way to illustrate the methods and to test the robustness of the conventional models.

Thus

$$
D_{j, t}^{+}=\left\{\begin{array}{l}
0, \text { if } \nabla Y_{j, t} \leq 0 \\
1, \text { if } \nabla Y_{j, t}>0
\end{array}\right.
$$

codes the arousal responses as 1 if there is a positive change in arousal and 0 otherwise. We will model the log odds of a positive change $\left(D_{j, t}^{+}=1\right)$ relative to no change or a negative change $\left(D_{j, t}^{+} \leq 0\right)$. A full trinomial model would require a bivariate model to be constructed for $D_{j, t}^{+}$and $D_{j, t}^{-}$as in (Liesenfeld, Nolte, and Pohlmeier 2006).

For modelling the probability of a positive change in arousal response we use regressors formed by taking lagged values of $\nabla I_{t}$, where $\mathrm{I}$ is acoustic intensity As in the ARMAX analysis of continuous responses given above let

$$
X_{t}=\nabla I_{t}
$$

and the vector of regressors at time $t$ is chosen to be

$$
x_{t}^{T}=\left(1, X_{t-1}, \ldots, X_{t-11}\right) .
$$

Note for the analysis of binary data we have selected lags 1 to 11, adding lag 11 because in some individuals it was significant. Instantaneous responses (Lag 0) were not significant for any of the 32 individual binary analyses.

We let $\mathcal{F}_{j, t-1}$ denote the information contained in the past binary response values $\left\{D_{j, s}^{+}: s<t\right\}$ and in the past and present in $x_{t}^{T}$. We assume that the conditional probability of a positive change occuring at time $t$ is

$$
\pi_{j, t}=P\left(D_{j, t}^{+}=1 \mid \mathcal{F}_{j, k, t-1}\right)
$$


and denote the log odds of a positive change as

$$
W_{j, t}=\operatorname{logit}\left(\pi_{k, t}\right)=\log \left(\frac{\pi_{j, t}}{1-\pi_{j, t}}\right) .
$$

The class of observation driven models that we employ here assumes that this "state" variable is a linear combination in the past 11 changes in intensity, $x_{t}^{T}$, and a linear filter applied to past predictive responses. That is

$$
\begin{aligned}
W_{j, t} & =\beta_{j, 0}+\sum_{l=1}^{11} \beta_{j, l} \nabla I_{t-l}+Z_{j, t} \\
& =x_{t}^{T} \beta_{j}+Z_{j, t}
\end{aligned}
$$

with an "error" process of the autoregressive moving average type

$$
Z_{j, t}=\sum_{l=1}^{p_{j}} \phi_{l, j}\left(Z_{j, t}+e_{j, t-l}\right)+\sum_{l=1}^{q_{j}} \theta_{l, j} e_{j, t-l}
$$

where

$$
e_{j, t}=\frac{D_{j, t}^{+}-\pi_{k, t}}{\sqrt{\pi_{k, t}\left(1-\pi_{k, t}\right)}}
$$

are Pearson residuals. The information set used in the linear predictor $W_{j, t}$ could be expanded to include past values of negative sign changes as well as past changes in the size of the change in responses observed - see (Rydberg and Shephard 2003) for details.

Note that the $e_{j, t}$ are zero mean, unit variance martingale differences with respect to $\mathcal{F}_{j, t-1}$ and hence the expected value of the logit of the probability of $D_{j, t}^{+}$is linear in $\nabla I_{t-l}$ for $l=1, \ldots, 11$. Although these past differenced intensity values also enter (nonlinearly) into the predictive residuals they do not impact the expected value of the logit response to them.

Throughout this analysis we have found that pure autoregressive processes in which $q_{j}=0$ are adequate to model the serial dependence in the binary time series. In particular, when $p_{j}=1$ we have a simple geometrically decaying weighted average of past Pearson predictive residuals modifying the impact that current time regression variables $x_{t}^{T}$ have on the log odds of a positive response in arousal:

$$
Z_{j, t}=\phi_{1, j} \sum_{l=1}^{\infty} \phi_{1, j}^{l} e_{j, t-l}
$$

For instance, if $\phi_{1, j}>0$ (which is the case in all 32 individual listener series we have modelled) then there is positive serial dependence induced in the state variable $W_{j, t}$ which decays exponentially with increasing lag $l$. This form is termed a generalized linear autoregressive moving average model and has been extensively used particularly for Poisson counts - see (Benjamin, Rigby, and Stasinopoulos 2003) and (Davis, Dunsmuir, and Streett 2003) for instance. It is a member of the class of non-Gaussian, exponential family response observation driven state space models for time series. Alternatives are BARMA models (Wang and Li 2011) in which $e_{j, t}$ are replaced by the unscaled errors $D_{j, t}^{+}-\pi_{k, t}$. 
As an illustration of the binary model we apply it to the four selected cases given in Figure 2. The corresponding results for the transfer function coefficients and $1.96 \times$ s.e. are given in Figure 3. The title in each subgraph shows the group to which the listener belongs, "\%Flat" (the proportion of time points for which the change in arousal response is 0 ) and the $p$-value for the Wald test used to assess the overall significance of the 11 individual transfer function coefficients. The panel for listener 5 suggests that they have a significant response to changes in the musical intensity. The panel for listener 13 suggests that the 11 lag transfer function is significantly different from zero also. Listener 23 is one for which $50 \%$ of the time their arousal response does not change from one time to the next. Here the transfer function response is not significant and this listener might reasonably be classified as being non-responsive to changes in acoustic intensity. Listener 22 shows no change in arousal response from one time to the next $88 \%$ of the time and it might be thought that such a lack of change in response would also suggest non-responsiveness to changes in intensity. In fact, the transfer function is significant for this listener. Clearly it does not necessarily follow that an individual who changes their response infrequently from time to time is unresponsive to the intensity of the music. The lag at which the peak in responsiveness (as measured by size of transfer function coefficient) can also vary. Listener 5 appears to respond most strongly after a short to moderate lag where listener 22 responds later. Note that in the above model for an individual listener the intercept, transfer function coefficients and autoregressive lags and coefficients could take different values.

Comparing Figures 2 and 3 it can be seen that there are some differences but the overall impression is very similar in the ARMAX model for the changes (both positive and negative) in arousal response and in the binary model for positive changes. In particular, the transfer functions are significant for listeners 5, 13, and 22 for both models, the peak lag matches for each of these three cases. At least for these four cases (which span a wide range of percentage of time in stasis for the respondents) the binary model is supportive of the conclusions reached using the continuous response ARMAX approach.

We now turn to an assessment of the results for all 32 series and test whether the individuals have the same mean logit responsiveness, as represented by the transfer function coefficients $\beta_{1}, \ldots, \beta_{11}$, or differ either within groups or overall.

\subsection{Are there differences between individual listeners' responses?}

In this subsection we revisit the questions addressed in the main paper concerning variations in responsiveness between individual listeners. We first fit the above binary time series model to each individual listener's binary response series $D_{j, t}^{+}$. For this analysis we used a lag $P=1$ autoregressive specification for each individual series. The value of the autoregressive parameter $\phi_{1, j}$ was allowed to vary by series - indeed this was necessary since the hypothesis that this serial dependence was the same across all series was rejected. The combined likelihood under this fully saturated alternative has $32 \times 13=$ 416 parameters. We then refit the above model using the likelihood which combines all 32 individual likelihoods but places restrictions, reflecting a null hypothesis of interest, on the parameters across the component likelihoods. To test the null hypothesis that $\beta_{j}=\beta$ (a common value) for all $j$ versus the alternative that some individuals at least have differing responsiveness to changes in intensity, the likelihood under the null hypothesis was optimized over the 32 autoregressive parameters and the 12 parameters in the common $\beta$. The likelihood ratio test statistic was $G^{2}=713.4$ on 372 d.f. which is highly significant. Therefore individuals differ in their average log-odds of probability of a positive change in response to changes in the music's intensity. This test included a test that the intercepts were the same for all 32 respondents. If the null hypothesis is recast to 

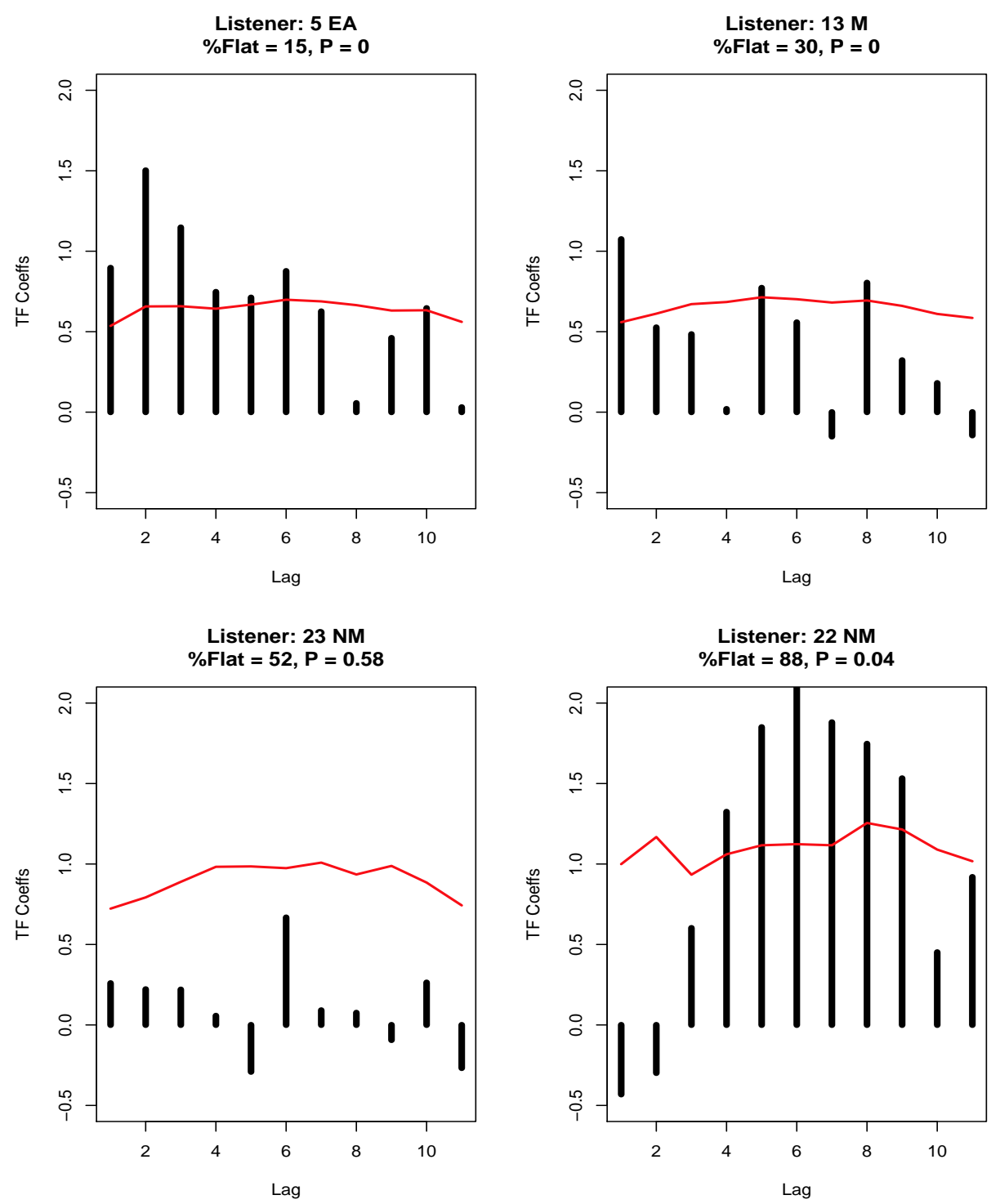

Figure 3. Transfer Function Coefficients Using the 11 lag unstructured TF Binary GLARMA model for positive responses $(1.96 \times$ standard errors for individual coefficients are shown as the red line.)

allow individuals to have differing intercept levels but restricts them all to have the same 11 lag transfer function coefficients then $G^{2}=453.8$ on 341 d.f., $p=4 \times 10^{-5}$. Again, even allowing for individual base level responsiveness to differ, there are highly significant differences between individual listener responses to changes in the music intensity.

Could this difference be due to differences between group average responses with the assumption being that individuals in each of the three groups have the same responsiveness? When the null hypothesis was framed as "individuals within each group had the same transfer function coefficients but these could differ between groups", that is

$$
W_{j t}=\beta_{G, 0}+\sum_{l=1}^{11} \beta_{G, l} X_{t-l}+Z_{j t}, j \in G=\mathrm{EA}, \mathrm{M}, \mathrm{NM},
$$

then $G^{2}=422.14$ on 319 d.f. with $p=9 \times 10^{-5}$. Hence individual differences cannot be ascribed to differences between group average responses alone. 
This conclusion is supported by the analysis of combined responses within groups by treating the number of positive changes observed in the group of listeners at each time $t$ as a binomial with number of trials given by the group size $m_{G}=8,8$, and 16 respectively for $G=\mathrm{EA}, \mathrm{M}$, and NM. Letting

$$
D_{G, t}^{+}=\sum_{j \in G} D_{j, t}^{+}
$$

we assume that

$$
D_{G, t}^{+} \sim \operatorname{Binomial}\left(m_{G}, \pi_{G, t}\right), G=\mathrm{EA}, \mathrm{M}, \mathrm{NM},
$$

where

$$
\operatorname{logit}\left(\pi_{G, t}\right)=W_{j, t}=\beta_{G, 0}+\sum_{l=1}^{11} \beta_{G, l} X_{t-l}+Z_{G t}, G=\mathrm{EA}, \mathrm{M}, \mathrm{NM}
$$

and where $Z_{G t}$ is an autoregression of degree $P_{G}$ in Pearson predictive residuals

$$
e_{G, t}=\frac{D_{G, t}^{+}-m_{G} \pi_{G, t}}{\sqrt{m_{G} \pi_{G, t}\left(1-\pi_{G, t}\right)}} .
$$

Note that in (1) each of the 32 individual series has its own $Z_{j, t}$ whereas, necessarily in the group binomial model (2), $Z_{G t}$ can only differ by group. The autoregressive degrees required were $P=2$ for the EA and $\mathrm{M}$ groups, and $P=3$ for the NM group. The above model was fit using our GLARMA software (available as R package glarma for download) but now with a binomial response distribution for each of the three groups as well as all individuals combined treated as a binomial with $m=32$. For all groups the transfer functions were individually significant at lags 1 through 10 and additionally at lag 11 for the NM groups and for all individuals combined. Some differences between the shapes and sizes of the group transfer functions can be observed. As was found for the analysis of continuous response transfer functions above, of the three groups the M group shows some tendency to differ from the EA and NM groups with the peak responsiveness occurring slightly earlier for the M group than for the other two. However these differences are not significant.

Three versions of (2) were compared as summarized in Table 1. Testing Model I (intercept and 11 lag transfer functions are the same across all 3 musical expertise groups) against Model III (all differ across groups) has $G^{2}=28.97$ on 24 d.f. and $p=0.22$ and Model I cannot be rejected. Allowing each group to have its own intercept but differing lag 11 transfer functions (Model II) gives $G^{2}=24.29$ on 22 d.f. $p=0.33$. In summary there are no significant differences between the average logit response across the three musician groups. This is a different conclusion than was reached for the analysis of group average continuous responses given in the main paper where it was found that the $\mathrm{M}$ group differed significantly from the EA and NM groups.

In summary, we find highly significant differences between individual listeners' propensities to respond with a positive change in arousal to changes in acoustic intensity, but that this is not due to differences in average or overall group responsiveness. In view of this we next delve more deeply into individual responsiveness with a view to describing the nature of these differences. 
Table 1. Results from fitting models to group binomial series of positive changes in arousal response.

\begin{tabular}{|llll|}
\hline Model & AIC & $-2 \log (L)$ & d.f. \\
\hline I: $\beta_{G, 0}, \ldots, \beta_{G, 11}$ same & 3675.5 & 3637.53 & 19 \\
II: $\beta_{G, 1}, \ldots, \beta_{G, 11}$ same & 3674.9 & 3632.85 & 21 \\
III: $\beta_{G, 0}, \ldots, \beta_{G, 11}$ differ & 3694.6 & 3608.56 & 43 \\
\hline
\end{tabular}

\subsection{Characterizing differences in individual listener responses}

The individual 11 lag transfer functions were obtained in the analysis of the last subsection and we now explore their features in more detail with the view of identifying different types of responsiveness across the individuals in the study.

The first question we consider is that of deciding if a listener is a "significant responder," as judged by the simplified binomial analysis. We identify a significant responder as one for whom the Wald test for the combined 11 lag transfer functions is significant at the $5 \%$ level. Of the 32 listeners, 12 were classified as significant responders. This may be contrasted with the 17 significant responders judged by the earlier continuous response ARMAX analysis.

Is the significance of the transfer function related to other factors such as degree of stasis in response or musical expertise measured by the OMSI score? To examine this we computed the logit of the Wald $p$-value and examined scatter plots and correlations of this with the OMSI score and \%Flat. The correlation with OMSI score is -0.26 but not significant $(p=0.16)$ and that with $\%$ Flat is 0.32 but only marginally significant $(p=0.07)$. While the significance level of the transfer function is not clearly related to OMSI score, the negative sign on the correlation is at least consistent with the idea that listeners who have higher OMSI score may have a higher chance of a significant response. Given that the Wald $p$-values for EA, M, and NM groups are almost identical, they show little sign of relationships with OMSI or \%Flats.

The unsmoothed and smoothed transfer functions for the non-significant and significant cases are shown in Figure 4. The thick light grey line in each panel shows the average for the curves in the panel. For the non-significant cases the mean is slightly positive with an upward bow around the middle lags but the values are considerable closer to zero than the average curves for significant cases. The transfer functions for significant cases are generally positive or have a positive peak. The transfer functions for non-significant cases tend to be negative or positive with a reasonably balanced scatter of lines above and below the zero line. For the 12 significant responses shown in the figure several peak at low to moderate lags and then either taper off or turn negative. Others peak for longer lags suggesting that these individuals are slower to respond to changes in acoustic intensity. Yet others have flat response profiles with no obvious peak over the 11 lags.

For the 12 listeners who demonstrated a significant response, we determined the lag at which the maximum value of the 11 lag transfer function was obtained as well as that for a 5 point triangular moving average smooth of it. The peak lags for the original and smoothed 11 lag transfer functions are strongly correlated $(0.74, p=0.01)$ as we might expect. Peak lags determined by either method are not significantly correlated with OMSI Score. Percentage flats (\%Flats) are positively but not significantly correlated with peak lag of the unsmoothed transfer function $(0.40, p=0.2)$ and with peak lag of the smoothed transfer function $(0.63, p=0.03)$. These observations, whilst weak and based on only 12 listeners, are consistent with the hypothesis that listeners who respond positively more frequently (low \%Flats) have an earlier peak in their responsiveness as was observed using standard ARMAX analysis discussed at the start of this section.

It is of interest to compare the results obtained using the standard ARMAX analysis with those obtained with the binary model. Of the 32 listeners, those 15 that did not 
11 Lag TF Non Sig Cases

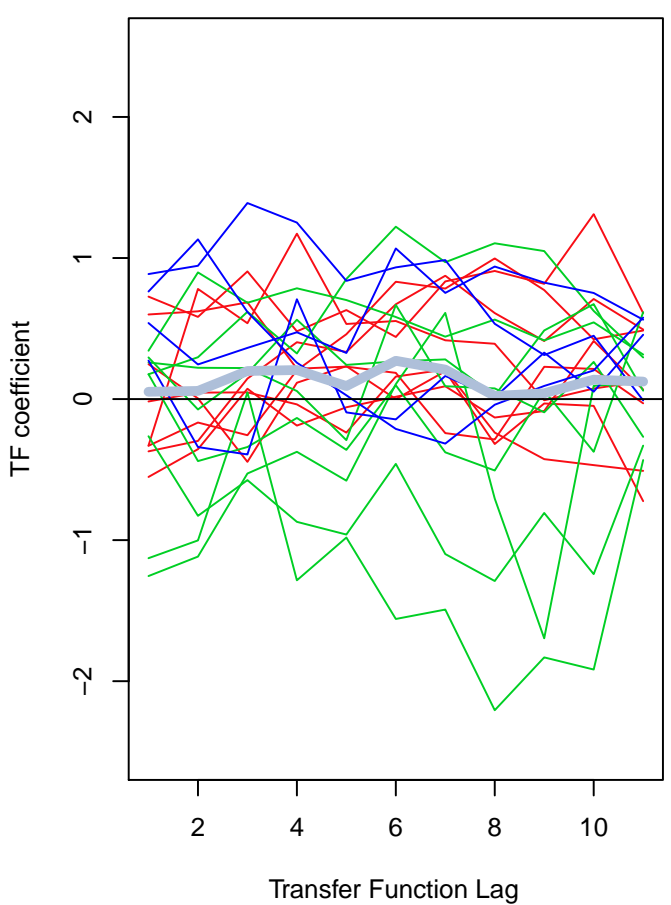

11 Lag TF Sig

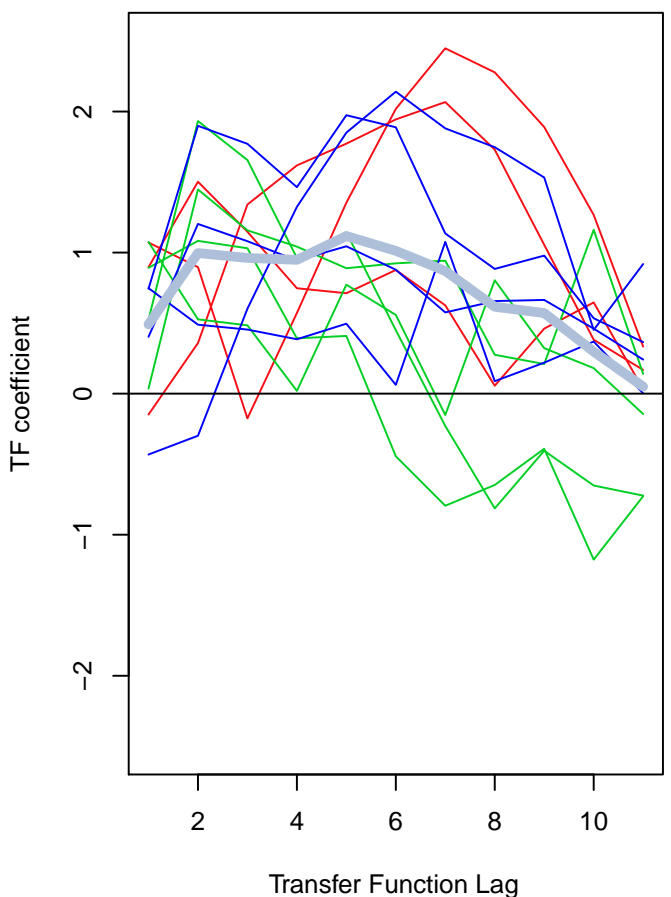

11 Lag TF Non Sig Cases

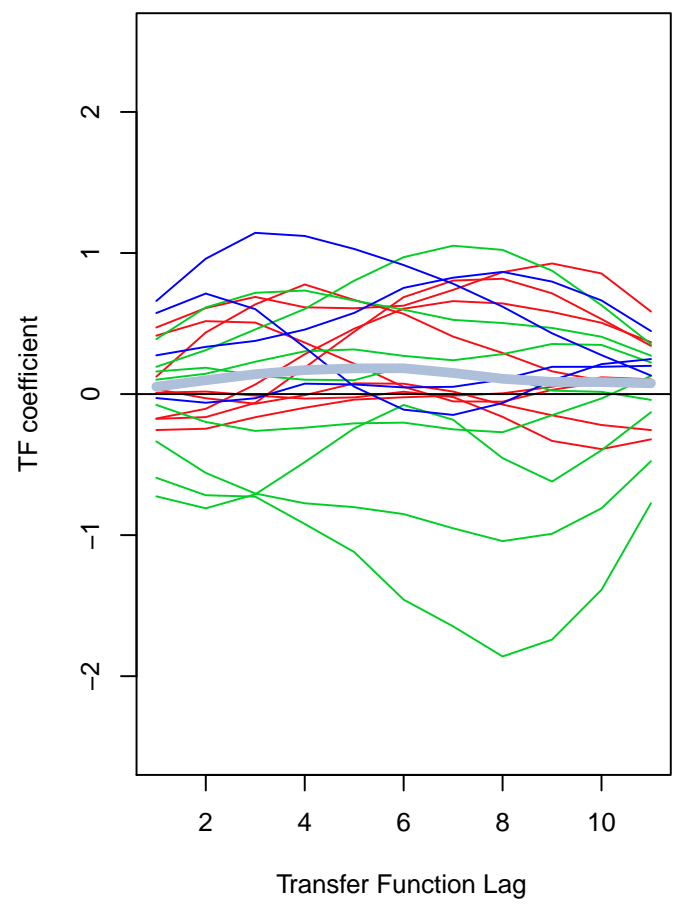

11 Lag TF Sig

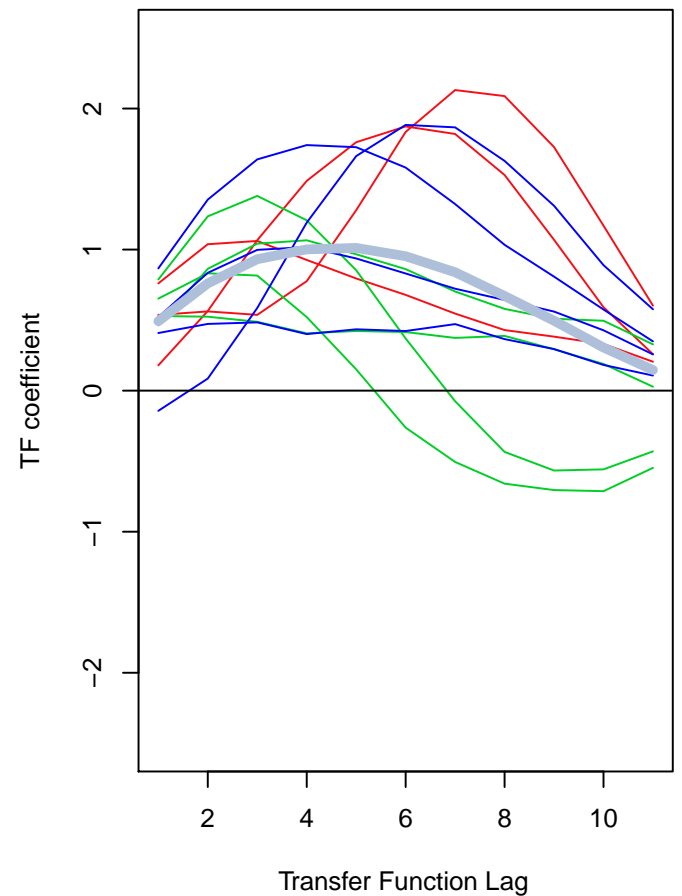

Figure 4. Transfer function coefficients for the individual binomial analyses, distinguishing the statistically significant and insignificant cases (see the text for details). The left hand pair of graphs are unsmoothed, the right hand pair smoothed. The thick light grey line on each graph is the mean of the other lines. 


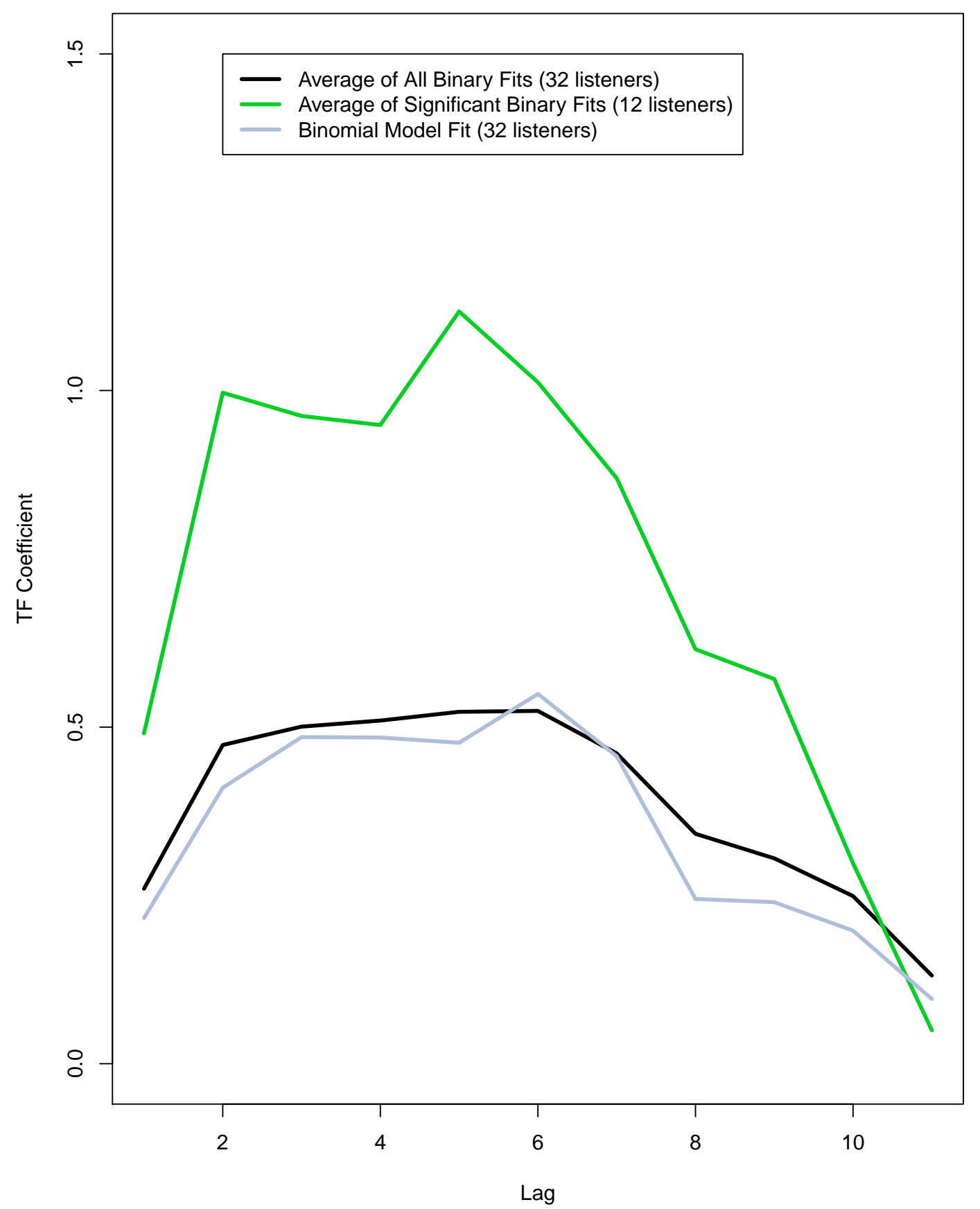

Figure 5. Transfer function coefficients for aggregated binomial series.

have $5 \%$ level significant overall Wald statistic for their ARMAX transfer functions were also non-significant for their positive change binary transfer function. Hence the binary model did not detect any additional significant listener responses. Of the 17 that had a significant ARMAX model transfer functions, 12 had a significant positive change binary transfer function and 5 did not. Therefore the ARMAX modelling detected significant responses in $50 \%$ more listeners than did the binary modelling. This is not necessarily 
inconsistent because the ARMAX model allows changes in response in both directions and takes into account size of response whereas the binary model considers only positive changes and disregards the size of change. Overall we conclude that the binary and ARMAX analyses are mutually coherent.

Furthermore ARMAX and binary models are roughly consistent in their indications of the lag at which peak responsiveness occurs, particularly bearing in mind that they are determined from unsmoothed transfer functions. Thus, of those 12 cases for which the binary model yielded an overall significant response the lag at which the maxima occurred matched the ARMAX-determined lag perfectly in 8 cases (listeners 4, 5, 8, 9, $10,13,21,22$ ), differed by one lag in 1 case (listener 28), by 3 lags in 2 cases (listeners 16 and 20) and by 6 lags for 1 case (listener 24). The cases where the difference was more than 1 lag were those for which the individual transfer functions were quite noisy in their structure. If the 17 cases for which the ARMAX transfer function is overall significant are considered, 10 matched the binomial-determined lag perfectly, 4 differed by 1 lag, 2 by 3 lags, 1 by 5 lags, and 1 by 6 lags. Thus the binary analysis is supportive of conclusions reached about timing of responsiveness obtained using what might have been inappropriate ARMAX analysis for individuals; the validity of the ARMAX analyses is thus supported.

Figure 5 compares the transfer function obtained by fitting to three single binomial series: that aggregating all 32 responses with a binomial response model, that averaging all individual binary fits and that averaging the 12 significant binary fits. The binomial fit to the aggregated 32 individual binary responses is similar to that obtained by averaging the transfer functions fit to all 32 responses. The average over the 12 significant fits has stronger transfer function coefficients which is the same as in the lower left panel of Figure 4.

\subsection{Conclusion from the binomial analyses}

The binary and binomial analyses presented here in general support the ARMAX analyses, in spite of the caveats raised, strongly confirming the variation between individuals in responsiveness to acoustic intensity changes. It is only in the case of the comparisons between listener groups, EA, M, and NM, that the binomial analysis is more equivocal than the continuous ARMAX analysis, and this is likely due to the reduction in data usage in the binomial approach to the group comparisons. In the companion paper (Dean, Bailes, and Dunsmuir 2014) comparing responses to four different musical extracts we therefore focus on the continuous ARMAX analyses.

\section{References}

Benjamin, Michael A., Robert A. Rigby, and D. Mikis Stasinopoulos. 2003. "Generalized Autoregressive Moving Average Models." Journal of the American Statistical Association 98 (461): 214-223. http: //pubs.amstat.org/doi/abs/10.1198/016214503388619238.

Davis, Richard A., William T. M. Dunsmuir, and Sarah B. Streett. 2003. "Observation-Driven Models for Poisson Counts." Biometrika 90 (4): 777-790. http://www.jstor.org/stable/30042088.

Dean, Roger T., Freya Bailes, and William T. M. Dunsmuir. 2014. "Shared and Distinct Mechanisms of Individual and Expertise-Group Perception of Expressed Arousal in Four Works." Journal of Mathematics and Music 8 (3): 207-223. http://dx.doi.org/10.1080/17459737.2014.928753.

Liesenfeld, Roman, Ingmar Nolte, and Winfried Pohlmeier. 2006. "Modelling Financial Transaction 
Price Movements: a Dynamic Integer Count Data Model." Empirical Economics 30 (4): 795-825. 10.1007/s00181-005-0001-1. http://dx.doi.org/10.1007/s00181-005-0001-1.

Rydberg, Tina Hviid, and Neil Shephard. 2003. "Dynamics of Trade-by-Trade Price Movements: Decomposition and Models." Journal of Financial Econometrics 1 (1): 2-25. http://jfec.oxfordjournals . org/content/1/1/2.abstract.

Wang, Chao, and Wai Keung Li. 2011. "On the Autopersistence Functions and the Autopersistence Graphs of Binary Autoregressive Time Series." Journal of Time Series Analysis 32 (6): 639-646. http://dx.doi.org/10.1111/j.1467-9892.2011.00721.x. 